\title{
Viscous Liquid Layer Sandwiched Between Two Generalized Thermoelastic Halfspaces
}

\author{
K. S. Harinath \\ Department of Mathematics, Bangalore University, Bangalore-560001
}

Received 3 June 1980

\begin{abstract}
A detailed investigation of waves in a viscous liquid layer of finite thickness sandwiched between two generalized thermoelastic halfspaces reveals the fact that for realistic situations one has to consider the effect of gravity at least in the liquid layer.
\end{abstract}

\section{Introduction}

This paper deals with waves in a viscous liquid layer sandwiched between two generalized thermoelastic halfspaces. We assume the presence of gravity in the liquid layer, in addition to viscosity. This paper is a continuation of the previous articles of the author ${ }^{1,2}$ and has immense applications to defence science, mainly in geophysical problems, such as water-covered or oil-covered layers in the earth's crust. It may also be noted that the problem considered here is more realistic ${ }^{3}$ than its elastic counterpart. The preliminaries on thermoelasticity may be found in Nowacki ${ }^{4}$. We refer to the earlier article $^{2}$ for the notation and terminology.

\section{Basic Equations}

We consider a liquid of density $\rho_{0}$ and of thickness $2 H$ sandwiched between two heat conducting homogeneous isotropic generalized thermoelastic halfspaces of densities $p_{1}$ and $\rho_{2}$. A rectangular cartesian coordinate system $(x, y, z)$ is set up in the media with the $z$-axis chosen downwards and the $x, y$ axes along the middle plane of the liquid, so that the interfaces correspond to $z=+H$ and $z=-H$ and for definiteness, the solid of density $p_{1}$, lies below the liquid layer, i.e. occupies the region $z \geqslant H$. The solids are assumed to be sufficiently incompressible with quite significant relaxation time factors, so that gravity effects may be ignored in the solid media, using a result of Jeffreys ${ }^{3}$. Moreover, the problem is converted into one of two dimensional plane strain by taking a plane section of the media containing the $x$ and $z$ axes and assuming independence of all quantities with reference to $y$. Before any disturbance (say, an explosion) the media are uniformly maintained at a constant temperature $T^{\circ}$. The 
displacement components $\left(u_{0}, o, w_{0}\right)$ in the liquid layer may be expressed in terms of a potential function $\chi$ by

$$
u_{0}=\frac{\partial \chi}{\partial x} \quad w_{0}=\frac{\partial \chi}{\partial z}
$$

where $\chi$ satisfies the partial differential equation

$$
\alpha_{0}^{2} \frac{\partial^{2} \chi}{\partial x^{2}}+\alpha_{0}^{2} \frac{\partial^{2} \chi}{\partial z^{2}}=\frac{\partial^{2} \chi}{\partial t^{2}}-g \frac{\partial \chi}{\partial z}
$$

in which $\alpha_{0}$ denotes the velocity of sound waves in the liquid, $g$ the acceleration due to gravity and $t$ the time variable.

To obtain progressive waves, a simple-harmonic time-dependence factor $\exp \{(\delta x-i \omega t)\}$ is assumed for $\chi$, where $\delta$ denotes the wave number and $\omega$ the frequency parameter. Then Eqn. (2) yields

$$
\alpha_{0}^{2} \frac{d^{2} \chi}{d z^{2}}+g \frac{d \chi}{d z}+\left(\omega^{2}-\delta^{2} \alpha_{0}^{2}\right) \chi=0
$$

which on solving leads to

$$
\chi=\left[A_{0} e^{-m_{0} z}+B_{0} e^{m_{0} z}\right] \exp \left\{-\frac{g z}{2 \alpha_{0}^{2}}+i(\delta x-\omega t)\right\}
$$

where

$$
m_{0}=\sqrt{\delta^{2}-\omega^{2} / \alpha_{0}^{2}+g^{2} / 4 \alpha_{0}^{4}}, \quad R e\left(m_{0}\right) \geqslant 0
$$

The normal and shear components of the stresses in the viscous liquid are given in terms of $\chi$ by

$$
\left.\begin{array}{l}
\sigma_{z z}^{\prime}=2 v \frac{\partial^{2} \chi}{\partial z^{2}}+v^{\prime}\left\{\frac{\partial^{2} \chi}{\partial x^{2}}+\frac{\partial^{2} \chi}{\partial z^{2}}\right\}+p_{0} \frac{\partial^{2} \chi}{\partial t^{2}} \\
\sigma_{x z}^{\prime}=2 v \frac{\partial^{2} \chi}{\partial x \partial z}
\end{array}\right\}
$$

where $\nu_{;} \nu^{\prime}$ denote the viscosity coefficients ${ }^{5}$.

For the solid halfspace $z \geqslant H$ of density $\rho_{1}$, the displacement components $\left(u_{1}, O, w_{1}\right)$ are given by

$$
u_{1}=\frac{\partial \phi_{1}}{\partial x}-\frac{\partial \psi_{1}}{\partial z}, \quad w_{1}=\frac{\partial \phi_{1}}{\partial z}+\frac{\partial \psi_{1}}{\partial x}
$$

where the potential functions $\phi_{1} . \psi_{1}$ and the temperature deviation $T_{1}$ from $T^{\circ}$ are given by the following expressions as in (Ref. 2)

$$
\begin{aligned}
& \left.\phi_{1}=\left[A_{1} e^{-z a_{1}}+B_{1} e^{-z b_{1}}\right] e^{i(\delta x-\omega t)}\right] \\
& \left.\psi_{1}=C_{1} e^{-z \sigma_{1}} e^{i(\delta x-\omega t)}\right\} \\
& \gamma_{1} \tau_{1} T_{1}=\rho_{1}\left[A_{1}\left(\omega^{2}-\alpha_{1}^{2} f_{1}^{2}\right) e^{-z a_{1}}+B_{1}\left(\omega^{2}-\alpha_{1}^{2} q_{1}^{2}\right) e^{-z b_{1}}\right] e^{i(\delta x-\Delta t)}
\end{aligned}
$$

which tend to zero as $z \rightarrow \infty$. 
In Eqn. (8), $\gamma_{1}$ is the ratio of the coefficient of thermal expansion to isothermal compressibility, $\tau_{1}=1-i \omega \tau_{1}^{\prime}$ where $\tau_{1}^{\prime}$ is the relaxation time factor, $a_{1}=\sqrt{\delta^{2}-f_{1}^{2}}$, $b_{1}=\sqrt{\delta^{2}-q_{1}^{2}}, c_{1}=\sqrt{\delta^{2}-\omega^{2} / \beta_{1}^{2}}, \operatorname{Re}\left(a_{1}\right), \operatorname{Re}\left(b_{1}\right), \operatorname{Re}\left(c_{1}\right)$ are all non-negative, $\alpha_{1}$ is the isothermal compressional wave velocity, $\beta_{1}$ is the shear wave velocity and $f_{1}^{2}, q_{1}^{2}$ are the roots of the biquadratic equation

$$
k_{1} \alpha_{1}^{2} \zeta^{4}-\left[k_{1} \omega^{2}+i \omega \rho_{1} s_{1} \tau_{1} \alpha_{1}^{2}\left(1+\epsilon_{1} \tau_{1}\right)\right] \zeta^{2}+i \omega^{3} \rho_{1} s_{1} \tau_{1}=0
$$

where $k_{1}$ is the coefficient of thermal conductivity, $s_{1}$ is the specific heat at constant strain and $\epsilon_{0}=\gamma_{1}^{2} T^{0} / s_{1} \rho_{1}^{2} \alpha_{1}^{2}$ is the coupling constant. $\quad\left(\epsilon_{1}\right.$ is of the order $10^{-2}$ while $\tau_{1}^{\prime}$ is of the order $10^{-11}$ ).

The normal and shear stresses in the solid media are given by the expressions

$$
\begin{aligned}
& \sigma_{z z}^{(1)}=\rho_{1} \alpha_{1}^{2}\left(\frac{\partial^{2} \phi_{1}}{\partial x^{2}}+\frac{\partial^{2} \phi_{1}}{\partial z^{2}}\right)+2 \rho_{1} \beta_{1}^{2}\left(\frac{\delta^{2} \psi_{1}}{\delta x \partial^{z}}-\frac{\partial^{2} \phi_{1}}{\partial x^{2}}\right)-\gamma_{1} \tau_{1} T_{1} \\
& \sigma_{x z}^{(1)}=\rho_{1} \beta_{1}^{2}\left(2 \frac{\partial^{2} \phi_{1}}{\partial x \partial z}+\frac{\partial^{2} \psi_{1}}{\partial x^{2}}-\frac{\partial^{2} \psi_{1}}{\partial z^{2}}\right)
\end{aligned}
$$

A similar analysis remains valid for the halfspace $z \leqslant-H$ of density $p_{2}$ above the liquid layer. To obtain the corresponding expressions, we have to merely replace the subscripts ' 1 ' in Eqn. (8) by subscripts ' 2 ' and change the sign of $z$ throughout to get

$$
\begin{aligned}
& \phi_{2}=\left[A_{2} e^{z a_{2}}+B_{2} e^{z b_{2}}\right] e^{i(\delta x-\omega t)} \\
& \psi_{2}=C_{2} e^{z o_{2}} e^{i(\delta x-\omega t)} \\
& \gamma_{2} \tau_{2} T_{2}=\rho_{2}\left[A_{2}\left(\omega^{2}-\alpha_{2}^{2} f_{2}^{2}\right) e^{z a_{2}}+B_{2}\left(\omega^{2}-\alpha_{2}^{2} q_{2}^{2}\right) e^{z b_{2}}\right] e^{i(\delta s-\omega t)}
\end{aligned}
$$

which tend to zero as $z \rightarrow-\infty$, with self-explanatory notations.

\section{Boundary Conditions}

In order to eliminate the eight unknowns occuring in the Eqns. (4), (8) and (11), namely, $A_{0}, B_{0}, A_{1}, B_{1}, C_{1}, A_{2}, B_{2}, C_{2}$, we impose the following natural conditions at the interfaces. 'The normal displacement, the normal stress, the tangential stress and the temperature deviation are all continuous'.

\section{The $\omega-\delta$ Equation}

This equation (also called the frequency equation or the dispersion relation) is obtained by equating the determinant of the eighth order of coefficients of the unknowns to zero. The eight equations satisfied by the eight unknowns $A_{0}, B_{0}, A_{1}, B_{1}, C_{1}, A_{2}$, $B_{2}, C_{2}$ are

$$
\begin{aligned}
& a_{1} e^{-H a_{1}} A_{1}+b_{1} e^{-H b_{1}} B_{1}-i \delta e^{-H c_{1} C_{1}}=a_{0} e^{-a_{0} H} A_{0}-b_{0} e^{b_{0} H} B_{0} \\
& a_{2} e^{H a_{2}} A_{2}+b_{2} e^{H b_{2}} B_{2}-i \delta e^{H a_{2}} C_{2}=a_{0} e^{a_{0} H} A_{0}-b_{0} e^{-b_{0} H} B_{0} \\
& \rho_{1} D_{1} e^{-H a_{1}} A_{1}+\rho_{1} D_{1} e^{-H b_{1}} B_{1}-2 i \delta \rho_{1} \beta_{1}^{2} c_{1} e^{-H c_{1}} C_{1}
\end{aligned}
$$




$$
\begin{aligned}
& =\left[\left(2 v+v^{\prime}\right) a_{0}^{2}-\rho_{0} \omega^{2}-v^{\prime} \delta^{2}\right] e^{-H a_{0}} A_{0} \\
& +\left[\left(2 v+v^{\prime}\right) b_{0}^{2}-\rho_{0} \omega^{2}-v^{\prime} \delta^{2}\right] e^{H b_{0}} B_{0} \\
& \mathrm{\rho}_{2} D_{2} e^{H a_{2}} A_{2}+\rho_{2} D_{2} e^{H b_{2}} B_{2}-2 i \delta \rho_{2} \beta_{2}^{2} c_{2} e^{H 0_{2}} C_{2} \\
& =\left[\left(2 v+v^{\prime}\right) a_{0}^{2}-\rho_{0} \omega^{2}-v^{\prime} \delta^{2}\right] e^{H a_{0}} A_{0}+\left[\left(2 v+v^{\prime}\right) b_{0}^{2}-\rho_{0} \omega^{2}\right. \\
& \left.-\nu^{\prime} \delta^{2}\right] e^{-H b} 0 B_{0} \\
& 2 i \delta p_{1} \beta_{1}^{2} a_{1} e^{-H a_{1}} A_{1}+2 i \delta \rho_{1} \beta_{1}^{2} b_{1} e^{-H b_{1} B_{1}}-\rho_{1} D_{1} e^{-H o_{1}} C_{1} \\
& =2 v i \delta a_{0} e^{-a_{0} H} A_{0}-2 v i \delta b_{0} e^{b_{0} H} B_{0} \\
& 2 i \delta \rho_{2} \beta_{2}^{2} a_{2} e^{H a_{2}} A_{2}+2 i \delta \rho_{2} \beta_{2}^{2} b_{2} e^{H b_{2}} B_{2}-\rho_{2} D_{2} e^{H c_{2}} C_{2} \\
& =2 v i \delta a_{0} e^{a_{0} H} A_{0}-2 v i \delta b_{0} e^{-b_{0} H_{B}} \\
& \left(\omega^{2}-\alpha_{1}^{2} f_{1}^{2}\right) e^{-H a_{1}} A_{1}+\left(\omega^{2}-\alpha_{1}^{2} q_{1}^{2}\right) e^{-H b_{1} B_{1}}=0 \\
& \left(\omega^{2}-\alpha_{2}^{2} f_{2}^{2}\right) e^{H a_{2}} A_{2}+\left(\omega^{2}-\alpha_{2}^{2} q_{2}^{2}\right) e^{H b_{2}} B_{2}=0
\end{aligned}
$$

where we have set

$$
\begin{array}{ll}
a_{0}=m_{0}+g / 2 \alpha_{0}^{2} & b_{0}=m_{0}-g / 2 \alpha_{0}^{2} \\
D_{1}=2 \beta_{1}^{2} \delta^{2}-\omega^{2} & D_{2}=2 \beta_{2}^{2} \delta^{2}-\omega^{2}
\end{array}
$$

Employing the notations

$$
\begin{aligned}
& \left(2 v+v^{\prime}\right) a_{0}^{2}-\rho_{0} \omega^{2}-v^{\prime} \delta^{2}=a^{*} \\
& \left(2 v+v^{\prime}\right) b_{0}^{2}-\rho_{0} \omega^{2}-v^{\prime} \delta^{2}=b^{*}
\end{aligned}
$$

the consistency criterion after simple manipulations is given by

$$
\begin{aligned}
& \Delta \equiv-\exp \left\{-\frac{g H}{\alpha_{0}^{2}}-H\left(a_{1}+b_{1}+c_{1}\right)+H\left(a_{2}+b_{2}+c_{2}\right)\right\} \\
& \begin{array}{ccccc}
a_{0} & b_{0} & a_{1} & b_{1} \\
a^{*} & -b^{*} & p_{1} D_{1} & p_{1} D_{1}
\end{array} \\
& \times \quad \begin{array}{cccc}
2 i \delta \vee a_{0} & 2 i \delta v b_{0} & 2 i \delta \rho_{1} \beta_{1}^{2} a_{1} & 2 i \delta \rho_{1} \beta_{1}^{2} b_{1} \\
0 & 0 & \omega^{2}-\alpha_{1}^{2} f_{1}^{2} & \omega^{2}-\alpha_{1}^{2} q_{1}^{2} \\
0 & 0 & 0 & 0 \\
a_{0} e^{2 a_{0} H} & b_{0} e^{-2 b_{0} H} & 0 & 0
\end{array} \\
& \begin{array}{llll}
a^{*} e^{2 a_{0} H} & -b^{*} e^{-2 b_{0} H} & 0 & 0
\end{array} \\
& 2 i \delta v a_{0} e^{2 a_{0} H} \quad 2 i \delta v b_{0} e^{2 b_{0} H} \quad 0 \quad 0 \\
& -i \delta \quad 0 \quad 0 \\
& \begin{array}{cccc}
-2 i \delta \rho_{1} \beta_{1}^{2} C_{1} & 0 & 0 & 0 \\
-\rho_{1} D_{1} & 0 & 0 & 0 \\
0 & 0 & 0 & 0 \\
0 & \omega^{2}-\alpha_{2}^{2} f_{2}^{2} & \omega^{2}-\alpha_{2}^{2} q_{2}^{2} & 0 \\
0 & a_{2} & b_{2} & -i \delta \\
0 & \rho_{2} D_{2} & \rho_{2} D_{2} & -2 i \delta \rho_{2} \beta_{2}^{2} c_{2} \\
0 & 2 i \delta \rho_{2} \beta_{2}^{2} a_{2} & 2 i \delta \rho_{2} \beta_{2}^{2} b_{2} & -\rho_{2} D_{2}
\end{array} \mid=0
\end{aligned}
$$


On further simplification Eqn. (14) becomes

$$
\begin{aligned}
\Delta \equiv & \rho_{1} \rho_{2} \exp \left\{H\left(a_{2}+b_{2}+c_{2}-a_{1}-b_{1}-c_{1}\right)\right\} \\
& \times\left[\left(\eta_{1} b_{0}+\xi_{1} b^{*}\right)\left(\eta_{2} a_{0}-\xi_{2} a^{*}\right) e^{2 m_{0} H}-\left(\eta_{1} a_{0}-\xi_{1} a^{*}\right)\left(\eta_{2} b_{0}+\xi_{2} b^{*}\right)\right. \\
& \left.e^{-2 m_{0} H}\right]=0
\end{aligned}
$$

where for $j=1,2$

$$
\begin{aligned}
\eta_{j}= & D_{j}\left(\rho_{j} D_{j}+2 \delta^{2} v\right) \alpha_{j}^{2}\left(q_{j}^{2}-f_{j}^{2}\right)+4 c_{j} \beta_{j}^{2} \delta^{2}\left(\rho_{j} \beta_{j}^{2}-\nu\right) \\
& \times\left[\omega^{2}\left(b_{j}-a_{j}\right)-\alpha_{j}^{2}\left(b_{j} f_{j}^{2}-a_{j} q_{j}^{2}\right)\right] \\
\xi_{j}= & \left(4 \beta_{j}^{2} \delta^{2}-\omega^{2}\right)\left[\omega^{2}\left(b_{j}-a_{j}\right)-\alpha_{j}^{2}\left(b_{j} f_{j}^{2}-a_{j} q_{j}^{2}\right)\right]
\end{aligned}
$$

Thus the consistency criterion is given by

$$
e^{2 m_{0} H}\left(\eta_{1} b_{0}+\xi_{1} b^{*}\right)\left(\eta_{2} a_{0}-\xi_{2} a^{*}\right)-e^{-2 m_{0} H}\left(\eta_{1} a_{0}-\xi_{1} a^{*}\right)\left(\eta_{2} b_{0}+\xi_{2} b^{*}\right)=0
$$

or

$$
\begin{aligned}
\tanh 2 m_{0} H= & \frac{-\left(\rho_{0} \omega^{2}+v^{\prime} \delta\right)^{2}\left(\xi_{1} \eta_{2}-\xi_{2} \eta_{1}\right) m_{0}-\left(2 v+v^{\prime}\right)\left(\xi_{1} \eta_{2}+\xi_{2} \eta_{1}\right)\left(m_{0}^{2}-\frac{g^{2}}{4 \alpha_{0}^{4}}\right)}{\eta_{1} \eta_{2}\left(m_{0}^{2}-\frac{g^{2}}{4 \alpha_{0}^{4}}\right)-\xi_{1} \xi_{2}\left(\rho_{0} \omega^{2}+v^{\prime} \delta^{2}\right)^{2}-\xi_{1} \xi_{2}\left(2 v+v^{\prime}\right)^{2}\left(m_{0}^{2}-\frac{g^{2}}{4 \alpha_{0}^{4}}\right)^{2}} \\
& +\xi_{1} \xi_{2}\left(2 v+v^{\prime}\right)\left(\rho_{0} \omega^{2}+v^{\prime} \delta^{2}\right)\left(2 m_{0}^{2}+\frac{g^{2}}{2 \alpha_{0}^{4}}\right) \\
& -\frac{g}{2 \alpha_{0}^{2}}\left\{\left(2 v+v^{\prime}\right)\left(\xi_{1} \eta_{2}-\xi_{2} \eta_{1}\right)\left(m_{0}^{2}-\frac{g^{2}}{4 \alpha_{0}^{4}}\right)\right. \\
& \left.+\left(\rho_{0} \omega^{2}+v^{\prime} \delta^{2}\right)\left(\xi_{1} \eta_{2}+\xi_{2} \eta_{1}\right)\right\}
\end{aligned}
$$

In the absence of viscous terms, the above Eqn. (17) reduces to

$$
\tanh 2 m_{0} H=\frac{\rho_{0} m_{0} \omega^{2}\left(\xi_{1} \eta_{2}-\xi_{2} \eta_{1}\right)}{\eta_{1} \eta_{2}\left(m_{0}^{2}-\frac{g^{2}}{4 \alpha_{0}^{4}}\right)-\rho_{0} \omega^{1} \xi_{1} \xi_{2}-\frac{g \rho_{0} \omega^{2}}{2 \alpha_{0}^{2}}\left(\xi_{1} \eta_{2}+\xi_{2} \eta_{1}\right)}
$$

If gravity terms are also omitted, then Eqn. (18) becomes

$$
\tanh 2 H \sqrt{\delta^{2}-\frac{\omega^{2}}{\alpha_{0}^{2}}}=\frac{\rho_{0} \omega^{2}\left(\xi_{1} \eta_{2}-\xi_{2} \eta_{1}\right) \sqrt{\delta^{2}-\omega^{2} / \alpha_{0}^{2}}}{\eta_{1} \eta_{2}\left(\delta^{2}-\omega^{2} / \alpha_{0}^{2}\right)-\rho_{0} \omega^{4} \xi_{1} \xi_{2}}
$$

which is similar to its elastic counterpart ${ }^{3}$.

\section{Conclusions}

Equation (17) generalizes the corresponding Eqn. (24) of (ref. 2; p. 141) and includes both the viscous as well as gravitational effects. Several limiting cases of the above 
Eqn. (17) may be analysed ${ }^{2}$. A theoretical analysis shows that in most of the expressions, the gravitational effects are quite pronounced. These expressions are more cumbersome than those in [ref. 2] and hence not repeated here. However, we list the following conclusions:

(1) As the frequency increases, the effect of viscosity becomes vanishingly small.

(2) The effect of viscosity is to slightly increase the phase-velocity of the propagated waves and also the attenuation in the $x$-direction.

(3) Very high frequencies or a very thin liquid layer lead to the same approximation of the dispersion relation which yields expressions for the viscosity coefficients $v$ and $v^{\prime}$. In such cases, the thermal terms may also be neglected.

(4) If the solids are incompressible, then in the case of a thin liquid layer, the effect of thermal terms may also be neglected in the case of small frequencies. i.e. for small frequencies, the compressibility of the solids do not play a significant role. Also, gravity effects may be ignored for small frequencies.

(5) Gravity effects are quite pronounced for large frequencies.

(6) In realistic situations, such as oil-covered layers in the earth's crust gravity terms must not be neglected. In order to verify this statement, we include the following expression, wherein the solids are incompressible.

$$
\begin{aligned}
\tanh 2 m_{0} H= & \frac{\frac{g \rho_{0} \delta \omega^{4}}{2 \alpha_{0}^{2}}\left\{4 \delta^{3}\left(\rho_{1} c_{1} \beta_{1}^{4}-\rho_{2} c_{2} \beta_{2}^{4}\right)+\rho_{1}\left(2 \delta^{2} \beta_{1}^{2}-\omega^{2}\right)^{2}-\rho_{2}\left(2 \delta^{2} \beta_{2}^{2}-\omega^{2}\right)^{2}\right\}}{\rho_{0}^{2} \rho_{2} \delta^{2} \omega^{8}-4 \delta^{4} \omega^{4} \rho_{0} m_{0}\left(\rho_{1} c_{1} \beta_{1}^{4}+\rho_{2} c_{2} \beta_{2}^{4}\right)-16 \delta^{6} \rho_{1} \rho_{2} c_{1} c_{2} \beta_{1}^{4} \beta_{2}^{4}\left(m_{0}^{2}-g^{2} / 4 \alpha_{0}^{4}\right)} \\
& -4 \delta^{3} \rho_{1} \rho_{2}\left(m_{0}^{2}-g^{2} / 4 \alpha_{0}^{4}\right)\left\{c_{1} \beta_{1}^{4}\left(2 \delta^{2} \beta_{1}^{2}-\omega^{2}\right)^{2}+c_{2} \beta_{2}^{4}\left(2 \delta^{2} \beta_{2}^{2}-\omega^{2}\right)^{2}\right\} \\
& -\rho_{0} m_{0} \delta \omega^{4}\left\{\rho_{1}\left(2 \delta^{2} \beta_{1}^{2}-\omega^{2}\right)^{2}+\rho_{2}\left(2 \delta^{2} \beta_{2}^{2}-\omega^{2}\right)^{2}\right\}-\rho_{1} \rho_{2}\left(m_{0}^{2}-\frac{g^{2}}{4 \alpha_{0}^{4}}\right) \\
& \times\left(2 \delta^{2} \beta_{1}^{2}-\omega^{2}\right)^{2}\left(2 \delta^{2} \beta_{2}^{2}-\omega_{s}^{2}\right)^{2}
\end{aligned}
$$

We notice that in Eqn. (20), the gravity effects are quite pronounced, as stated earlier.

(7) There exists dispersion of waves in all the cases.

The problem considered in this paper has immense applications to defence science, mainly, in geophysical problems, such as oil-covered layers in the earth's crust. The numerical study of this paper is under preparation.

\section{Referènces}

1. Harinath, K. S., Def. Sci. J., 27 (1977), 1.

2. Harinath, K. S. \& Muthuswamy, V. P., Def. Sci. J., 28 (1978), 137.

3. Ewing, W. M., Jardetzky, W. S. \& Press, F., 'Elastic Waves in layered media', (Mc Graw-Hill), 1957.

4. Nowacki, W., 'Thermoelasticity', (Polish Scientific Publishers), 1962.

5. Segal, L. A., 'Mathematics applied to continuum mechanics', (Macmillan Press), 1977. 